\title{
Nonlocal Problems for Fractional Differential Equations via Resolvent Operators
}

\author{
Zhenbin Fan ${ }^{1}$ and Gisèle Mophou ${ }^{2}$ \\ ${ }^{1}$ Department of Mathematics, Changshu Institute of Technology, Suzhou, Jiangsu 215500, China \\ ${ }^{2}$ Laboratoire CEREGMIA, Université des Antilles et de la Guyane, Campus Fouillole, \\ Guadeloupe (FWI) 97159 Pointe-à-Pitre, France
}

Correspondence should be addressed to Gisèle Mophou; gmophou@univ-ag.fr

Received 7 April 2013; Accepted 15 May 2013

Academic Editor: Sotiris Ntouyas

Copyright (C) 2013 Z. Fan and G. Mophou. This is an open access article distributed under the Creative Commons Attribution License, which permits unrestricted use, distribution, and reproduction in any medium, provided the original work is properly cited.

We discuss the continuity of analytic resolvent in the uniform operator topology and then obtain the compactness of Cauchy operator by means of the analytic resolvent method. Based on this result, we derive the existence of mild solutions for nonlocal fractional differential equations when the nonlocal item is assumed to be Lipschitz continuous and neither Lipschitz nor compact, respectively. An example is also given to illustrate our theory.

\section{Introduction}

In this paper, we are concerned with the existence of mild solutions for a fractional differential equation with nonlocal conditions of the form

$$
\begin{gathered}
D^{\alpha} u(t)=A u(t)+J_{t}^{1-\alpha} f(t, u(t)), \quad 0<t \leq b, \\
u(0)=u_{0}-g(u),
\end{gathered}
$$

where $D^{\alpha}$ is the Caputo fractional derivative of order $\alpha$ with $0<\alpha<1, A: D(A) \subset X \rightarrow X$ is the infinitesimal generator of a resolvent $S_{\alpha}(t), t \geq 0, X$ is a real Banach space endowed with the norm $\|\cdot\|$, and $f$ and $g$ are appropriate continuous functions to be specified later.

The theory of fractional differential equations has received much attention over the past twenty years, since they are important in describing the natural models such as diffusion processes, stochastic processes, finance, and hydrology. Many notions associated with resolvent are developed such as integral resolvent, solution operators, $\alpha$-resolvent operator functions, $(a, k)$-regularized resolvent, and $\alpha$-order fractional semigroups. All of these notions play a central role in the study of Volterra equations, especially the fractional differential equations. Concerning the literature, we refer the reader to the books $[1,2]$, the recent papers [3-20], and the references therein.

On the other hand, abstract differential equations with nonlocal conditions have also been studied extensively in the literature, since it is demonstrated that the nonlocal problems have better effects in applications than the classical ones. It was Byszewski and Lakshmikantham [21] who first studied the existence and uniqueness of mild solutions for nonlocal differential equations. And the main difficulty in dealing with the nonlocal problem is how to get the compactness of solution operator at zero, especially when the nonlocal item is only assumed to be Lipschitz continuous or continuous. Many authors developed different techniques and methods to solve this problem. For more details on this topic, we refer to $[10,11,22-33]$ and references therein.

In this paper, we combine the above two directions and study the nonlocal fractional differential equation (1) governed by operator $A$ generating an analytic resolvent. A standard approach in deriving the mild solution of (1) is to define the solution operator $Q$. Then, conditions are given such that some fixed point theorems such as Browder's and Schauder's fixed point theorems can be applied to get a fixed point for solution operator $Q$, which gives rise to a mild solution of (1). The key step of using this approach is 
to prove the compactness of Cauchy operator $G$ associated with solution operator $Q$. When the operator $A$ generates a compact semigroup, it is well known that the Cauchy operator $G$ is also compact. However, to the best of our knowledge, it is unknown when $A$ generates a compact resolvent. The main difficulty of this problem lies in the fact that there is no property of semigroups for resolvent.

To this end, we will first discuss the continuity of resolvent in the uniform operator topology in this paper. In fact, we prove that the compact analytic resolvent is continuous in the uniform operator topology. Based on this result, we can prove the compactness of Cauchy operator. As a consequence, we obtain the existence of mild solutions for (1) when the nonlocal item is Lipschitz continuous. At the same time, we also derive the existence of mild solutions for (1) without the Lipschitz or compact assumption on the nonlocal item $g$ by using the techniques developed in [24, 30]. Actually, we only assume that $g$ is continuous on $C([0, b], X)$ and $g$ is completely determined on $[\delta, b]$ for some small $\delta>0$ or $g$ is continuous on $C([0, b], X)$ with $L^{1}([0, b], X)$ topology (see Corollaries 17-19).

This paper has four sections. In Section 2, we recall some definitions on Caputo fractional derivatives, analytic resolvent, and mild solutions to (1). In Section 3, we prove the compactness of Cauchy operator. Finally, in Section 4 we establish the existence of mild solutions of (1) when the nonlocal item satisfies different conditions. An example is also given in this section.

\section{Preliminaries}

Throughout this paper, let $b>0$ be fixed, and let $\mathbb{N}, \mathbb{R}$, and $\mathbb{R}_{+}$be the set of positive integers, real numbers, and nonnegative real numbers, respectively. We denote by $X$ the Banach space with the norm $\|\cdot\|, C([0, b], X)$ the space of all $X$-valued continuous functions on $[0, b]$ with the norm $\|u\|=\sup \{\|u(t)\|, t \in[0, b]\}$, and $L^{p}([0, b], X)$ the space of $X$ valued Bochner integrable functions on $[0, b]$ with the norm $\|f\|_{L^{p}}=\left(\int_{0}^{b}\|f(t)\|^{p} \mathrm{~d} t\right)^{1 / p}$, where $1 \leq p<\infty$. Also, we denote by $\mathscr{L}(X)$ the space of bounded linear operators from $X$ into $X$ endowed with the norm of operators.

Now, let us recall some basic definitions and results on fractional derivative and fractional differential equations.

Definition 1 (see [1]). The fractional order integral of the function $f \in L^{1}([0, b], X)$ of order $\alpha>0$ is defined by

$$
J_{t}^{\alpha} f(t)=\frac{1}{\Gamma(\alpha)} \int_{0}^{t}(t-s)^{\alpha-1} f(s) \mathrm{d} s,
$$

where $\Gamma$ is the Gamma function.

Definition 2 (see [1]). The Riemann-Liouville fractional order derivative of order $\alpha$ of a function $f \in L^{1}([0, b], X)$ given on the interval $[0, b]$ is defined by

$$
D_{L}^{\alpha} f(t)=\frac{1}{\Gamma(n-\alpha)} \frac{d^{n}}{d t^{n}} \int_{0}^{t}(t-s)^{n-\alpha-1} f(s) \mathrm{d} s,
$$

where $\alpha \in(n-1, n], n \in \mathbb{N}$.
Definition 3 (see [1]). The Caputo fractional order derivative of order $\alpha$ of a function $f \in L^{1}([0, b], X)$ given on the interval $[0, b]$ is defined by

$$
D^{\alpha} f(t)=\frac{1}{\Gamma(n-\alpha)} \int_{0}^{t}(t-s)^{n-\alpha-1} f^{(n)}(s) \mathrm{d} s,
$$

where $\alpha \in(n-1, n], n \in \mathbb{N}$.

In the remainder of this paper, we always suppose that $0<$ $\alpha<1$ and $A$ is a closed and densely defined linear operator on $X$.

Definition 4. A family $\left\{S_{\alpha}(t)\right\}_{t \geq 0} \subseteq \mathscr{L}(X)$ of bounded linear operators in $X$ is called a resolvent (or solution operator) generating by $A$ if the following conditions are satisfied:

(S1) $S_{\alpha}(t)$ is strong continuous on $\mathbb{R}_{+}$and $S_{\alpha}(0)=I$;

(S2) $S_{\alpha}(t) D(A) \subseteq D(A)$ and $A S_{\alpha}(t) x=S_{\alpha}(t) A x$ for all $x \in$ $D(A)$ and $t \geq 0$

(S3) the resolvent equation holds

$S_{\alpha}(t) x=x+\int_{0}^{t} g_{\alpha}(t-s) A S_{\alpha}(s) x \mathrm{~d} s \quad \forall x \in D(A), t \geq 0$.

For $\omega, \theta \in \mathbb{R}$, let

$$
\sum(\omega, \theta):=\{\lambda \in \mathbb{C}:|\arg (\lambda-\omega)|<\theta\} .
$$

Definition 5. A resolvent $S_{\alpha}(t)$ is called analytic, if the function $S_{\alpha}(\cdot): \mathbb{R}_{+} \rightarrow \mathscr{L}(X)$ admits analytic extension to a sector $\sum\left(0, \theta_{0}\right)$ for some $0<\theta_{0} \leq \pi / 2$. An analytic resolvent $S_{\alpha}(t)$ is said to be of analyticity type $\left(\omega_{0}, \theta_{0}\right)$ if for each $\theta<\theta_{0}$ and $\omega>\omega_{0}$ there is $M_{1}=M_{1}(\omega, \theta)$ such that $\|S(z)\| \leq M_{1} e^{\omega \operatorname{Re} z}$ for $z \in \sum(0, \theta)$, where $\operatorname{Re} z$ denotes the real part of $z$.

Definition 6. A resolvent $S_{\alpha}(t)$ is called compact for $t>0$ if for every $t>0, S_{\alpha}(t)$ is a compact operator.

According to Proposition 1.2 in [2], we can give the following definition of mild solutions for (1).

Definition 7. A function $u \in C([0, b], X)$ is called a mild solution of fractional evolution equation (1) if it satisfies

$$
\begin{aligned}
u(t)= & S_{\alpha}(t)\left[u_{0}-g(u)\right] \\
& +\int_{0}^{t} S_{\alpha}(t-s) f(s, u(s)) \mathrm{d} s, \quad 0 \leq t \leq b
\end{aligned}
$$

for every $u_{0} \in X$.

Next, we introduce the Hausdorff measure of noncompactness $\beta(\cdot)$ defined on each bounded subset $\Omega$ of Banach space $Y$ by

$$
\beta(\Omega)=\inf \{\varepsilon>0 ; \Omega \text { has a finite } \varepsilon \text {-net in } Y\} .
$$

Some basic properties of $\beta(\cdot)$ are given in the following lemma. 
Lemma 8 (see [34]). Let $Y$ be a real Banach space and let $B, C \subseteq Y$ be bounded; then the following properties are satisfied:

(1) $B$ is precompact if and only if $\beta(B)=0$;

(2) $\beta(B)=\beta(\bar{B})=\beta(\operatorname{conv} B)$, where $\bar{B}$ and conv $B$ mean the closure and convex hull of $B$, respectively;

(3) $\beta(B) \leq \beta(C)$ when $B \subseteq C$;

(4) $\beta(B+C) \leq \beta(B)+\beta(C)$, where $B+C=\{x+y ; x \in$ $B, y \in C\}$;

(5) $\beta(B \cup C) \leq \max \{\beta(B), \beta(C)\}$;

(6) $\beta(\lambda B)=|\lambda| \beta(B)$ for any $\lambda \in \mathbb{R}$;

(7) if the map $Q: D(Q) \subseteq Y \rightarrow Z$ is Lipschitz continuous with constant $k$, then $\beta(Q B) \leq k \beta(B)$ for any bounded subset $B \subseteq D(Q)$, where $Z$ is a Banach space.

The map $Q: W \subseteq Y \rightarrow Y$ is said to be a $\beta$-contraction if there exists a positive constant $k<1$ such that $\beta(Q C) \leq$ $k \beta(C)$ for any bounded closed subset $C \subseteq W$, where $Y$ is a Banach space.

Lemma 9 ([34], Darbo-Sadovskii). If $W \subseteq Y$ is bounded closed and convex, the continuous map $Q: W \rightarrow W$ is a $\beta$-contraction, then the map $Q$ has at least one fixed point in $W$.

\section{Compactness of Cauchy Operators}

Let Cauchy operator $G: C([0, b], X) \rightarrow C([0, b], X)$ be defined by

$$
(G f)(t)=\int_{0}^{t} S_{\alpha}(t-s) f(s) \mathrm{d} s, \quad t \in[0, b] .
$$

If $S_{\alpha}(t)$ is a compact $C_{0}$-semigroup, it is well known that $G$ is compact. However, it is unknown in case of compact resolvent. The main difficulty is that the resolvent does not have the property of semigroups. Thus, it seems to be more complicated to prove the compactness of Cauchy operator. Here, we will first discuss the continuity of resolvent in the uniform operator topology. Then, we can give the positive answer to the above problem.

In the remainder of this paper, we always assume that $M=\sup _{t \in[0, b]}\left\|S_{\alpha}(t)\right\|<+\infty$.

Lemma 10. Suppose $S_{\alpha}(t)$ is a compact analytic resolvent of analyticity type $\left(\omega_{0}, \theta_{0}\right)$. Then the following are hold:

(i) $\lim _{h \rightarrow 0}\left\|S_{\alpha}(t+h)-S_{\alpha}(t)\right\|=0$ for $t>0$;

(ii) $\lim _{h \rightarrow 0^{+}}\left\|S_{\alpha}(t+h)-S_{\alpha}(h) S_{\alpha}(t)\right\|=0$ for $t>0$;

(iii) $\lim _{h \rightarrow 0^{+}}\left\|S_{\alpha}(t)-S_{\alpha}(h) S_{\alpha}(t-h)\right\|=0$ for $t>0$.
Proof. (i) Let $S_{\alpha}(t)$ be an analytic resolvent of analyticity type $\left(\omega_{0}, \theta_{0}\right)$, and let $t>0$ be given. Then, by means of Cauchy integral formula, we have

$$
S_{\alpha}^{\prime}(t)=\frac{1}{2 \pi i} \int_{|z-t|=t \sin \theta} \frac{S_{\alpha}(z)}{(z-t)^{2}} \mathrm{~d} z
$$

Thus, for any $\omega>\omega_{0}, 0<\theta<\theta_{0}$, there exists a constant $M_{1}=M_{1}(\omega, \theta)$ such that

$$
\begin{aligned}
\left\|S_{\alpha}^{\prime}(t)\right\| & \leq M_{1}(2 \pi)^{-1} \int_{-\pi}^{\pi} e^{\omega(t+t \sin \theta \cos \gamma)}(t \sin \theta)^{-1} \mathrm{~d} \gamma \\
& \leq M_{1} e^{2 \omega t}(t \sin \theta)^{-1} .
\end{aligned}
$$

Now, let $\|x\| \leq 1$ and $|h|<t$. It follows from (11) that there exists a constant $M^{\prime}$ such that

$$
\begin{aligned}
\left\|S_{\alpha}(t+h) x-S_{\alpha}(t) x\right\| \\
\quad=\left\|\int_{t}^{t+h} S_{\alpha}^{\prime}(s) x \mathrm{~d} s\right\| \\
\quad \leq M^{\prime}\|x\|\left|\int_{t}^{t+h} s^{-1} \mathrm{~d} s\right| \\
\quad \leq M^{\prime}|\ln (t+h)-\ln t| \longrightarrow 0 \text { as } h \longrightarrow 0,
\end{aligned}
$$

which implies that $S_{\alpha}(t)$ is continuous in the uniform operator topology for $t>0$, that is,

$$
\lim _{h \rightarrow 0}\left\|S_{\alpha}(t+h)-S_{\alpha}(t)\right\|=0 \quad \text { for } t>0 .
$$

(ii) Let $\|x\| \leq 1, t>0$, and $\varepsilon>0$ be given. Since $S_{\alpha}(t)$ is compact, the set $W_{t}:=\left\{S_{\alpha}(t) x:\|x\| \leq 1\right\}$ is also compact. Thus, there exists a finite family $\left\{S_{\alpha}(t) x_{1}\right.$, $\left.S_{\alpha}(t) x_{2}, \ldots, S_{\alpha}(t) x_{m}\right\} \subset W_{t}$ such that for any $x$ with $\|x\| \leq 1$, there exists $x_{i}(1 \leq i \leq m)$ such that

$$
\left\|S_{\alpha}(t) x-S_{\alpha}(t) x_{i}\right\| \leq \frac{\varepsilon}{3(M+1)} \text {. }
$$

From the strong continuity of $S_{\alpha}(t), t \geq 0$, there exists $0<$ $h_{1}<\min \{t, b\}$ such that

$$
\left\|S_{\alpha}(t) x_{i}-S_{\alpha}(h) S_{\alpha}(t) x_{i}\right\| \leq \frac{\varepsilon}{3}, \quad \forall 0 \leq h \leq h_{1}, 1 \leq i \leq m .
$$

On the other hand, from (i), there exists $0<h_{2}<\min \{t, b\}$ such that

$$
\left\|S_{\alpha}(t+h) x-S_{\alpha}(t) x\right\| \leq \frac{\varepsilon}{3}, \quad \forall 0 \leq h \leq h_{2},\|x\| \leq 1
$$


Thus, for $0 \leq h \leq \min \left\{h_{1}, h_{2}\right\}$ and $\|x\| \leq 1$, it follows from (14)-(16) that

$$
\begin{aligned}
\| S_{\alpha}( & +h) x-S_{\alpha}(h) S_{\alpha}(t) x \| \\
\leq & \left\|S_{\alpha}(t+h) x-S_{\alpha}(t) x\right\| \\
& +\left\|S_{\alpha}(t) x-S_{\alpha}(t) x_{i}\right\| \\
& +\left\|S_{\alpha}(t) x_{i}-S_{\alpha}(h) S_{\alpha}(t) x_{i}\right\| \\
& +\left\|S_{\alpha}(h) S_{\alpha}(t) x_{i}-S_{\alpha}(h) S_{\alpha}(t) x\right\| \\
\leq & \left\|S_{\alpha}(t+h) x-S_{\alpha}(t) x\right\| \\
& +(M+1)\left\|S_{\alpha}(t) x-S_{\alpha}(t) x_{i}\right\| \\
& +\left\|S_{\alpha}(t) x_{i}-S_{\alpha}(h) S_{\alpha}(t) x_{i}\right\| \\
\leq & \frac{\varepsilon}{3}+\frac{\varepsilon}{3}+\frac{\varepsilon}{3} \\
\leq & \varepsilon
\end{aligned}
$$

which implies that $\lim _{h \rightarrow 0^{+}}\left\|S_{\alpha}(t+h)-S_{\alpha}(h) S_{\alpha}(t)\right\|=0$ for all $t>0$.

(iii) Let $t>0$ and $0<h<\min \{t, b\}$. Then, there exists $M>0$ such that

$$
\begin{aligned}
& \left\|S_{\alpha}(t)-S_{\alpha}(h) S_{\alpha}(t-h)\right\| \\
& \leq\left\|S_{\alpha}(t)-S_{\alpha}(t+h)\right\|+\left\|S_{\alpha}(t+h)-S_{\alpha}(h) S_{\alpha}(t)\right\| \\
& \quad+\left\|S_{\alpha}(h) S_{\alpha}(t)-S_{\alpha}(h) S_{\alpha}(t-h)\right\| \\
& \leq\left\|S_{\alpha}(t)-S_{\alpha}(t+h)\right\|+\left\|S_{\alpha}(t+h)-S_{\alpha}(h) S_{\alpha}(t)\right\| \\
& \quad+M\left\|S_{\alpha}(t)-S_{\alpha}(t-h)\right\| .
\end{aligned}
$$

It follows from (i) and (ii) that

$$
\lim _{h \rightarrow 0^{+}}\left\|S_{\alpha}(t)-S_{\alpha}(h) S_{\alpha}(t-h)\right\|=0 \quad \text { for } t>0 .
$$

This completes the proof.

Lemma 11. Suppose $S_{\alpha}(t)$ is a compact analytic resolvent of analyticity type $\left(\omega_{0}, \theta_{0}\right)$. Then Cauchy operator $G$ defined by (9) is a compact operator.

Proof. We will show $G: C([0, b], X) \rightarrow C([0, b], X)$ is compact by using the Arzela-Ascoli theorem. Let $D_{l}=$ $\{f \in C([0, b], X):\|f\| \leq l\}$ be any bounded subset of $C([0, b], X)$.
First, we claim that the set $G D_{l}$ is equicontinuous on $C([0, b], X)$. In fact, let $0 \leq t_{1} \leq t_{2} \leq b$ and $f \in D_{l}$; then we have

$$
\begin{aligned}
I:= & \left\|(G f)\left(t_{2}\right)-(G f)\left(t_{1}\right)\right\| \\
= & \left\|\int_{0}^{t_{2}} S_{\alpha}\left(t_{2}-s\right) f(s) \mathrm{d} s-\int_{0}^{t_{1}} S_{\alpha}\left(t_{1}-s\right) f(s) \mathrm{d} s\right\| \\
\leq & \int_{0}^{t_{1}}\left\|S_{\alpha}\left(t_{2}-s\right)-S_{\alpha}\left(t_{1}-s\right)\right\|\|f(s)\| \mathrm{d} s \\
& +\int_{t_{1}}^{t_{2}}\left\|S_{\alpha}\left(t_{2}-s\right) f(s)\right\| \mathrm{d} s \\
\leq & l \int_{0}^{t_{1}}\left\|S_{\alpha}\left(t_{2}-s\right)-S_{\alpha}\left(t_{1}-s\right)\right\| \mathrm{d} s+M l\left(t_{2}-t_{1}\right) .
\end{aligned}
$$

If $t_{1}=0$, it is easy to see that

$$
\lim _{t_{2} \rightarrow 0} I=0 \text {, uniformly for } f \in D_{l} \text {. }
$$

If $0<t_{1}<b$, for $0<\delta<t_{1}$, we have

$$
\begin{aligned}
I \leq & l \int_{0}^{t_{1}-\delta}\left\|S_{\alpha}\left(t_{2}-s\right)-S_{\alpha}\left(t_{1}-s\right)\right\| \mathrm{d} s \\
& +l \int_{t_{1}-\delta}^{t_{1}}\left\|S_{\alpha}\left(t_{2}-s\right)-S_{\alpha}\left(t_{1}-s\right)\right\| \mathrm{d} s+M l\left(t_{2}-t_{1}\right) \\
\leq & b l \sup _{s \in\left[0, t_{1}-\delta\right]}\left\|S_{\alpha}\left(t_{2}-s\right)-S_{\alpha}\left(t_{1}-s\right)\right\| \\
& +2 M l \delta+M l\left(t_{2}-t_{1}\right) .
\end{aligned}
$$

Note that from Lemma 10(i), we know $S_{\alpha}(t)$ is operator norm continuous uniformly for $t \in[\delta, b]$. Combining this and the arbitrariness of $\delta$ with the above estimation on $I$, we can conclude that

$$
\lim _{\left|t_{1}-t_{2}\right| \rightarrow 0} I=0, \quad \text { uniformly for } f \in D_{l} \text {. }
$$

Thus, $G D_{l}$ is equicontinuous on $C([0, b], X)$.

Next, we will show that the set $\left\{(G f)(t): f \in D_{l}\right\}$ is precompact in $X$ for every $t \in[0, b]$. It is easy to see that the set $\left\{(G f)(0): f \in D_{l}\right\}$ is precompact in $X$. Now, let $0<t \leq b$ be given and $0<\varepsilon<t$. Then

$$
\left\{S_{\alpha}(\varepsilon) \int_{0}^{t-\varepsilon} S_{\alpha}(t-s-\varepsilon) f(s) \mathrm{d} s: f \in D_{l}\right\}
$$


is precompact since $S_{\alpha}(\varepsilon)$ is compact. Moreover, for arbitrary $\varepsilon<\delta<t$, we have

$$
\begin{aligned}
& \left\|S_{\alpha}(\varepsilon) \int_{0}^{t-\varepsilon} S_{\alpha}(t-s-\varepsilon) f(s) \mathrm{d} s-\int_{0}^{t-\varepsilon} S_{\alpha}(t-s) f(s) \mathrm{d} s\right\| \\
& \leq l \int_{0}^{t-\varepsilon}\left\|S_{\alpha}(\varepsilon) S_{\alpha}(t-s-\varepsilon)-S_{\alpha}(t-s)\right\| \mathrm{d} s \\
& \leq l \int_{0}^{t-\delta}\left\|S_{\alpha}(\varepsilon) S_{\alpha}(t-s-\varepsilon)-S_{\alpha}(t-s)\right\| \mathrm{d} s \\
& \quad+l \int_{t-\delta}^{t-\varepsilon}\left\|S_{\alpha}(\varepsilon) S_{\alpha}(t-s-\varepsilon)-S_{\alpha}(t-s)\right\| \mathrm{d} s \\
& \leq l \int_{0}^{t-\delta}\left\|S_{\alpha}(\varepsilon) S_{\alpha}(t-s-\varepsilon)-S_{\alpha}(t-s)\right\| \mathrm{d} s+l \delta\left(M^{2}+M\right) .
\end{aligned}
$$

From Lemma 10(iii), we know

$$
\begin{array}{r}
S_{\alpha}(\varepsilon) S_{\alpha}(t-s-\varepsilon)-S_{\alpha}(t-s) \longrightarrow 0, \\
\text { as } \varepsilon \longrightarrow 0 \text { for } s \in[0, t-\delta] .
\end{array}
$$

Then, it follows from the Lebesgue dominated convergence theorem and the arbitrariness of $\delta$ that

$$
\begin{gathered}
\lim _{\varepsilon \rightarrow 0} \| S_{\alpha}(\varepsilon) \int_{0}^{t-\varepsilon} S_{\alpha}(t-s-\varepsilon) f(s) \mathrm{d} s \\
-\int_{0}^{t-\varepsilon} S_{\alpha}(t-s) f(s) \mathrm{d} s \|=0 .
\end{gathered}
$$

On the other hand,

$$
\begin{aligned}
& \left\|S_{\alpha}(\varepsilon) \int_{0}^{t-\varepsilon} S_{\alpha}(t-s-\varepsilon) f(s) \mathrm{d} s-\int_{0}^{t} S_{\alpha}(t-s) f(s) \mathrm{d} s\right\| \\
& \leq \| S_{\alpha}(\varepsilon) \int_{0}^{t-\varepsilon} S_{\alpha}(t-s-\varepsilon) f(s) \mathrm{d} s \\
& \quad-\int_{0}^{t-\varepsilon} S_{\alpha}(t-s) f(s) \mathrm{d} s \| \\
& \quad+\left\|\int_{0}^{t-\varepsilon} S_{\alpha}(t-s) f(s) \mathrm{d} s-\int_{0}^{t} S_{\alpha}(t-s) f(s) \mathrm{d} s\right\| \\
& \leq \| S_{\alpha}(\varepsilon) \int_{0}^{t-\varepsilon} S_{\alpha}(t-s-\varepsilon) f(s) \mathrm{d} s \\
& \quad-\int_{0}^{t-\varepsilon} S_{\alpha}(t-s) f(s) \mathrm{d} s \|+l \varepsilon M .
\end{aligned}
$$

Thus,

$$
\begin{gathered}
\lim _{\varepsilon \rightarrow 0} \| S_{\alpha}(\varepsilon) \int_{0}^{t-\varepsilon} S_{\alpha}(t-s-\varepsilon) f(s) \mathrm{d} s \\
-\int_{0}^{t} S_{\alpha}(t-s) f(s) \mathrm{d} s \|=0,
\end{gathered}
$$

which implies that $\left\{(G f)(t): f \in D_{l}\right\}$ is precompact in $X$ by using the total boundedness, and therefore $G$ is compact in view of Arzela-Ascoli theorem.

\section{Nonlocal Problems}

In this section, we always assume that $u_{0} \in X$ and that the operator $A$ generates a compact analytic resolvent $S_{\alpha}(t)$ of analyticity type $\left(\omega_{0}, \theta_{0}\right)$, and we will prove the existence of mild solutions of (1) when the nonlocal item $g$ is assumed to be Lipschitz continuous and neither Lipschitz nor compact, respectively.

Let $r$ be a fixed positive real number and

$$
W_{r}:=\{u \in C([0, b], X):\|u\| \leq r\} .
$$

Clearly, $W_{r}$ is a bounded closed and convex set. We make the following assumptions.

(H1) $f:[0, b] \times X \rightarrow X$ is continuous.

(H2) $g: C([0, b], X) \rightarrow X$ is Lipschitz continuous with Lipschitz constant $k$ such that $M k<1$.

Under these assumptions, we can prove the first main result in this paper.

Theorem 12. Assume that conditions (H1) and (H2) are satisfied. Then the nonlocal problem (1) has at least one mild solution provided that

$$
M\left[\left\|u_{0}\right\|+\sup _{u \in W_{r}}\|g(u)\|+b \sup _{s \in[0, b], u \in W_{r}}\|f(s, u(s))\|\right] \leq r .
$$

Proof. We consider the solution operator $Q: C([0, b], X) \rightarrow$ $C([0, b], X)$ defined by

$$
\begin{aligned}
(Q u)(t) & =S_{\alpha}(t)\left[u_{0}-g(u)\right]+\int_{0}^{t} S_{\alpha}(t-s) f(s, u(s)) \mathrm{d} s \\
& :=\left(Q_{1} u\right)(t)+\left(Q_{2} u\right)(t), \quad t \in[0, b] .
\end{aligned}
$$

It is easy to see that the fixed point of $Q$ is the mild solution of nonlocal Cauchy problem (1). Subsequently, we will prove that $Q$ has a fixed point by using Lemma 9 (Darbo-Sadovskii's fixed point theorem).

Firstly, we prove that the mapping $Q$ is continuous on $C([0, b], X)$. For this purpose, let $\left\{u_{n}\right\}_{n \geq 1}$ be a sequence in $C([0, b], X)$ with $\lim _{n \rightarrow \infty} u_{n}=u$ in $C([0, b], X)$. Then

$$
\begin{aligned}
& \left\|Q u_{n}-Q u\right\| \\
& \leq M\left[\left\|g\left(u_{n}\right)-g(u)\right\|+b \sup _{s \in[0, b]}\left\|f\left(s, u_{n}(s)\right)-f(s, u(s))\right\|\right] .
\end{aligned}
$$

By the continuity of $f$ and $g$, we deduce that $Q$ is continuous on $C([0, b], X)$. 
Secondly, we claim that $Q W_{r} \subseteq W_{r}$, where $W_{r}$ is defined by (30). In fact, for any $u \in W_{r}$, by (31), we have

$$
\begin{aligned}
\|Q u\| & \leq M\left(\left\|u_{0}\right\|+\|g(u)\|+\int_{0}^{b}\|f(s, u(s))\| \mathrm{d} s\right) \\
& \leq M\left(\left\|u_{0}\right\|+\sup _{u \in W_{r}}\|g(u)\|+b \sup _{s \in[0, b], u \in W_{r}}\|f(s, u(s))\|\right) \\
& \leq r
\end{aligned}
$$

which implies that $Q$ maps $W_{r}$ into itself.

Now, according to Lemma 9, it remains to prove that $Q$ is a $\beta$-contraction in $W_{r}$. From condition $(\mathrm{H} 2)$, we get that $Q_{1}$ : $W_{r} \rightarrow C([0, b], X)$ is Lipschitz continuous with constant $M k$. In fact, for $u, v \in W_{r}$, by (H2), we have

$$
\left\|Q_{1} u-Q_{1} v\right\| \leq M\|g(u)-g(v)\| \leq M k\|u-v\| .
$$

Thus, it follows from Lemma 8-(30) that $\beta\left(Q_{1} W_{r}\right) \leq$ $M k \beta\left(W_{r}\right)$.

For operator $Q_{2}: W_{r} \rightarrow C([0, b], X)$, we have $Q_{2} u=$ $G f_{u}$, where $f_{u}(\cdot)=f(\cdot, u(\cdot))$ is continuous on $[0, b]$ and $G$ is the Cauchy operator defined by (9). Thus, in view of Lemma 11, we know that $Q_{2}$ is compact on $C([0, b], X)$, and hence $\beta\left(Q_{2} W_{r}\right)=0$. Consequently,

$$
\beta\left(Q W_{r}\right) \leq \beta\left(Q_{1} W_{r}\right)+\beta\left(Q_{2} W_{r}\right) \leq M k \beta\left(W_{r}\right) .
$$

Since $M k<1$, the mapping $Q$ is a $\beta$-contraction on $W_{r}$. By Darbo-Sadovskii's fixed point theorem, the operator $Q$ has a fixed point in $W_{r}$, which is just the mild solution of nonlocal Cauchy problem (1).

Now, we give the following technical condition on function $g$.

(H) $g: C([0, b], X) \rightarrow X$ is continuous, and the set $g\left(\overline{\operatorname{conv}} Q W_{r}\right)$ is precompact, where $\overline{\operatorname{conv}} B$ denotes the convex closed hull of set $B \subseteq C([0, T], X)$, and $Q$ is given by (32).

Remark 13. It is easy to see that condition $(\mathrm{H})$ is weaker than the compactness and convexity of $g$. The same hypothesis can be seen from $[24,30]$, where the authors considered the existence of mild solutions for semilinear nonlocal problems of integer order when $A$ is a linear, densely defined operator on $X$ which generates a $C_{0}$-semigroup. After the proof of our main results, we will give some special types of nonlocal item $g$ which is neither Lipschitz nor compact, but satisfies the condition $(\mathrm{H})$ in the next Corollaries.

Theorem 14. Assume that conditions (H1) and (H) are satisfied. Then the nonlocal problem (1) has at least one mild solution provided that (31) holds.

Proof. We will prove that $Q$ has a fixed point by using Schauder's fixed point theorem. According to the proof of Theorem 12, we have proven that $Q: W_{r} \rightarrow W_{r}$ is continuous. Next, we will prove that there exists a set $W \subseteq W_{r}$ such that $Q: W \rightarrow W$ is compact.

For this purpose, let $0<t \leq b$ and $0<\delta<t$. It is easy to see that the set

$$
\left\{S_{\alpha}(\delta) S_{\alpha}(t-\delta)\left[u_{0}-g(u)\right]: u \in W_{r}\right\}
$$

is precompact since $S_{\alpha}(\delta)$ is compact. On the other hand, by Lemma 10(iii), we obtain

$$
\begin{aligned}
\lim _{\delta \rightarrow 0} & \| S_{\alpha}(t)\left[u_{0}-g(u)\right] \\
& -S_{\alpha}(\delta) S_{\alpha}(t-\delta)\left[u_{0}-g(u)\right] \|=0, \quad \forall u \in W_{r},
\end{aligned}
$$

which implies that $Q_{1} W_{r}(t)$ is precompact in $X$ by using the total boundedness. Next, we claim $Q_{1} W_{r}$ is equicontinuous on $[\eta, b]$ for any small positive number $\eta$. In fact, for $u \in W_{r}$ and $\eta \leq t_{1} \leq t_{2} \leq b$, we have

$$
\begin{aligned}
& \left\|\left(Q_{1} u\right)\left(t_{2}\right)-\left(Q_{1} u\right)\left(t_{1}\right)\right\| \\
& \leq\left\|S_{\alpha}\left(t_{2}\right)-S_{\alpha}\left(t_{1}\right)\right\|\left(\left\|u_{0}\right\|+\|g(u)\|\right) .
\end{aligned}
$$

By Lemma 10(i), $S_{\alpha}(t)$ is operator norm continuous for $t>$ 0 . Thus $S_{\alpha}(t)$ is operator norm continuous uniformly for $t \epsilon$ $[\eta, b]$, and hence

$$
\begin{array}{r}
\lim _{\left|t_{2}-t_{1}\right| \rightarrow 0}\left\|\left(Q_{1} u\right)\left(t_{2}\right)-\left(Q_{1} u\right)\left(t_{1}\right)\right\|=0, \\
\text { uniformly for } u \in W_{r},
\end{array}
$$

that is, $Q_{1} W_{r}$ is equicontinuous on $[\eta, b]$. Note that $Q_{2}$ is compact by Lemma 11. Therefore, we have proven that $Q W_{r}(t)$ is precompact for every $t \in(0, b]$ and $Q W_{r}$ is equicontinuous on $[\eta, b]$ for any small positive number $\eta$.

Now, let $W=\overline{\text { conv }} Q W_{r}$, we get that $W$ is a bounded closed and convex subset of $C([0, b], X)$ and $Q W \subseteq W$. It is easy to see that $Q W(t)$ is precompact in $X$ for every $t \in(0, b]$ and $Q W$ is equicontinuous on $[\eta, b]$ for any small positive number $\eta$. Moreover, we have that $g(W)=g\left(\overline{\operatorname{conv}} Q W_{r}\right)$ is precompact due to condition $(\mathrm{H})$.

Thus, we can now claim that $Q: W \rightarrow W$ is compact. In fact, it is easy to see that $Q_{1} W(0)=u_{0}-g(W)$ is precompact since $g(W)=g\left(\overline{\operatorname{conv}} Q W_{r}\right)$ is precompact. It remains to prove that $Q_{1} W$ is equicontinuous on $[0, b]$. To this end, let $u \in W$ and $0 \leq t_{1}<t_{2} \leq b$; then we have

$$
\left\|\left(Q_{1} u\right)\left(t_{2}\right)-\left(Q_{1} u\right)\left(t_{1}\right)\right\| \leq\left\|S_{\alpha}\left(t_{2}\right)-S_{\alpha}\left(t_{1}\right)\right\|\left\|u_{0}-g(u)\right\| .
$$

In view of the compactness of $g(W)$ and the strong continuity of $S_{\alpha}(t)$ on $[0, b]$, we obtain the equicontinuous of $Q_{1} W$ on $[0, b]$. Thus, $Q_{1}: W \rightarrow C([0, b], X)$ is compact by ArzelaAscoli theorem, and hence $Q: W \rightarrow W$ is also compact. Now, Schauder's fixed point theorem implies that $Q$ has a fixed point on $W$, which gives rise to a mild solution of nonlocal problem (1).

The following theorem is a direct consequence of Theorem 14. 
Theorem 15. Assume that conditions $(\mathrm{H} 1)$ and $(\mathrm{H})$ are satisfied for each $r>0$. If

$$
\begin{gathered}
\frac{\|g(u)\|}{\|u\|} \longrightarrow 0, \quad\|u\| \longrightarrow \infty, \\
\frac{\|f(t, x)\|}{\|x\|} \longrightarrow 0, \quad\|x\| \longrightarrow \infty
\end{gathered}
$$

uniformly for $t \in[0, b]$, then the nonlocal problem (1) has at least one mild solution.

Remark 16. It is easy to see that if there exist constants $L_{1}$, $L_{2}>0$ and $\gamma_{1}, \gamma_{2} \in[0,1)$ such that

$$
\begin{gathered}
\|g(u)\| \leq L_{1}(1+\|u\|)^{\gamma_{1}}, \\
\|f(t, x)\| \leq L_{2}(1+\|x\|)^{\gamma_{2}}
\end{gathered}
$$

for $t \in[0, b]$, then conditions (42) are satisfied.

Next, we will give special types of nonlocal item $g$ which is neither Lipschitz nor compact, but satisfies condition $(\mathrm{H})$.

We give the following assumptions.

(H3) $g: C([0, b], X) \rightarrow X$ is a continuous mapping which maps $W_{r}$ into a bounded set, and there is a $\delta=\delta(r) \epsilon$ $(0, b)$ such that $g(u)=g(v)$ for any $u, v \in W_{r}$ with $u(s)=v(s), s \in[\delta, b]$.

(H4) $g:\left(C([0, b], X),\|\cdot\|_{L^{1}}\right) \rightarrow X$ is continuous.

Corollary 17. Assume that conditions (H1) and (H3) are satisfied. Then the nonlocal problem (1) has at least one mild solution on $[0, b]$ provided that (31) holds.

Proof. Let

$$
\begin{aligned}
\left(Q W_{r}\right)_{\delta}= & \{u \in C([0, b], X) ; u(t)=v(t) \text { for } t \in[\delta, b], \\
& \left.u(t)=u(\delta) \text { for } t \in[0, \delta), \text { where } v \in Q W_{r}\right\} .
\end{aligned}
$$

From the proof of Theorem 14, we know that $\left(Q W_{r}\right)_{\delta}$ is precompact in $C([0, b], X)$. Moreover, by condition (H3), $g\left(\overline{\operatorname{conv}} Q W_{r}\right)=g\left(\overline{\operatorname{conv}}\left(Q W_{r}\right)_{\delta}\right)$ is also precompact in $C([0, b], X)$. Thus, all the hypotheses in Theorem 14 are satisfied. Therefore, there is at least one mild solution of nonlocal problem (1).

Corollary 18. Let condition (H1) be satisfied. Suppose that $g(u)=\sum_{j=1}^{p} c_{j} u\left(t_{j}\right)$, where $c_{j}$ are given positive constants, and $0<t_{1}<t_{2}<\cdots<t_{p} \leq b$. Then the nonlocal problem (1) has at least one mild solution on $[0, b]$ provided that

$$
M\left[\left\|u_{0}\right\|+\sum_{j=1}^{p} c_{j} r+b \sup _{s \in[0, b], u \in W_{r}}\|f(s, u(s))\|\right] \leq r .
$$

Proof. It is easy to see that the mapping $g$ with $g(u)=$ $\sum_{j=1}^{p} c_{j} u\left(t_{j}\right)$ satisfies condition (H3). And all the conditions in Corollary 17 are satisfied. So the conclusion holds.
Corollary 19. Assume that conditions (H1) and (H4) are satisfied. Then the nonlocal problem (1) has at least one mild solution on $[0, b]$ provided that (31) holds.

Proof. According to Theorem 14, it is sufficient to prove that the hypothesis $(\mathrm{H})$ is satisfied. For arbitrary $\epsilon>0$, there exists $0<\delta<b$ such that $\int_{0}^{\delta}\|u(s)\| \mathrm{d} s<\epsilon$ for all $u \in Q W_{r}$. Let

$$
\begin{array}{r}
\left(Q W_{r}\right)_{\delta}=\{u \in C([0, b], X) ; u(t)=v(t) \text { for } t \in[\delta, b], \\
\left.u(t)=u(\delta) \text { for } t \in[0, \delta), \text { where } v \in Q W_{r}\right\} .
\end{array}
$$

From the proof of Theorem 14, we know that $\left(Q W_{r}\right)_{\delta}$ is precompact in $C([0, b], X)$, which implies that $\left(Q W_{r}\right)_{\delta}$ is precompact in $L^{1}([0, b], X)$. Thus, $Q W_{r}$ is precompact in $L^{1}([0, b], X)$ as it has an $\epsilon$-net $\left(Q W_{r}\right)_{\delta}$. By condition $g$ : $\left(C([0, b], X),\|\cdot\|_{L^{1}}\right) \rightarrow X$ is continuous and $\overline{c o n v} Q W_{r} \subseteq$ $(L) \overline{\mathrm{Conv}} \mathrm{Q} W_{r}$, it follows that condition $(\mathrm{H})$ is satisfied, where $(L) \overline{\operatorname{conv}} B$ denotes the convex and closed hull of $B$ in $L^{1}([0, b], X)$. Therefore, the nonlocal problem (1) has at least one mild solution on $[0, b]$.

Finally, we give a simple example to illustrate our theory.

Example 20. Consider the following fractional partial differential heat equation in $\mathbb{R}$ :

$$
\begin{gathered}
D_{t}^{\alpha} w(t, x) \\
=\frac{\partial^{2} w(t, x)}{\partial x^{2}} \\
\quad+J_{t}^{1-\alpha} F(t, w(t, x)), \quad 0<x<1,0<t<1, \\
w(t, 0)=w(t, 1), \quad w_{x}^{\prime}(t, 0)=w_{x}^{\prime}(t, 1), \quad 0 \leq t \leq 1, \\
w(0, x)=w_{0}(x)-\sum_{j=1}^{p} c_{j} w\left(t_{j}, x\right), \quad 0<x<1,0<t_{j} \leq 1,
\end{gathered}
$$

where $0<\alpha<1$ and $D_{t}^{\alpha}$ denotes the Caputo fractional derivative.

We introduce the abstract frame as follows. Let $X=\{u \in$ $C([0,1], \mathbb{R}): u(0)=u(1)\}$. Let $A$ be the linear operator in $X$ defined by $D(A)=\left\{u \in X: u^{\prime}, u^{\prime \prime} \in X\right\}$ and for $u \in D(A), A u=u^{\prime \prime}$. Then, it is well known that $A$ generates a compact $C_{0}$ semigroup $T(t)$ for $t>0$ on $X$. From the subordination principle [6, Theorems 3.1 and 3.3], $A$ also generates a compact resolvent $S_{\alpha}(t)$ for $t>0$.

Assume that $f:[0,1] \times X \rightarrow X$ is a continuous function defined by $f(t, z)(x)=F(t, z(x)), 0 \leq x \leq 1$ and $g: C([0,1], X) \rightarrow X$ is also a continuous function defined by $g(u)(x)=w_{0}(x)-\sum_{j=1}^{p} c_{j} u\left(t_{j}\right)(x), 0 \leq t, x \leq 1$, where $u(t)(x):=w(t, x)$. 
Under these assumptions, the fractional partial differential heat equation (47) can be reformulated as the abstract problem (1). If the inequality

$$
M\left[\left\|w_{0}\right\|+\sum_{j=1}^{p} c_{j} r+\sup _{s \in[0,1], u \in W_{r}}\|f(s, u(s))\|\right] \leq r
$$

holds for some constant $r>0$, there exists at least one mild solution for fractional equation (47) in view of Corollary 18.

\section{Acknowledgments}

This work was completed when the first author visited the Pennsylvania State University. The author is grateful to Professor Alberto Bressan and the Department of Mathematics for their hospitality and providing good working conditions. The work was supported by the NSF of China (11001034) and Jiangsu Overseas Research \& Training Program for University Prominent Young \& Middle-aged Teachers and Presidents.

\section{References}

[1] I. Podlubny, Fractional Differential Equations, vol. 198 of Mathematics in Science and Engineering, Academic Press, San Diego, Calif, USA, 1999.

[2] J. Prüss, Evolutionary Integral Equations and Applications, vol. 87 of Monographs in Mathematics, Birkhäuser, Basel, Switzerland, 1993.

[3] R. P. Agarwal, Y. Zhou, and Y. He, "Existence of fractional neutral functional differential equations," Computers \& Mathematics with Applications, vol. 59, no. 3, pp. 1095-1100, 2010.

[4] R. P. Agarwal, M. Belmekki, and M. Benchohra, "Existence results for semilinear functional differential inclusions involving Riemann-Liouville fractional derivative," Dynamics of Continuous, Discrete \& Impulsive Systems, vol. 17, no. 3, pp. 347-361, 2010.

[5] R. P. Agarwal, V. Lakshmikantham, and J. J. Nieto, "On the concept of solution for fractional differential equations with uncertainty," Nonlinear Analysis, vol. 72, no. 6, pp. 2859-2862, 2010.

[6] E. Bajlekova, Fractional evolution equations in Banach spaces [Ph.D. thesis], University Press Facilities, Eindhoven University of Technology, 2001.

[7] M. Benchohra, J. Henderson, S. K. Ntouyas, and A. Ouahab, "Existence results for fractional order functional differential equations with infinite delay," Journal of Mathematical Analysis and Applications, vol. 338, no. 2, pp. 1340-1350, 2008.

[8] M. Benchohra and B. A. Slimani, "Existence and uniqueness of solutions to impulsive fractional differential equations," Electronic Journal of Differential Equations, no. 10, pp. 1-11, 2009.

[9] M. Fečkan, J. Wang, and Y. Zhou, "Controllability of fractional functional evolution equations of Sobolev type via characteristic solution operators," Journal of Optimization Theory and Applications, vol. 156, no. 1, pp. 79-95, 2013.

[10] E. Hernández, D. O’Regan, and K. Balachandran, “On recent developments in the theory of abstract differential equations with fractional derivatives," Nonlinear Analysis, vol. 73, no. 10, pp. 3462-3471, 2010.
[11] E. Hernández, D. O’Regan, and K. Balachandran, "Existence results for abstract fractional differential equations with nonlocal conditions via resolvent operators," Indagationes Mathematicae, vol. 24, no. 1, pp. 68-82, 2013.

[12] C. Lizama, "Regularized solutions for abstract Volterra equations," Journal of Mathematical Analysis and Applications, vol. 243, no. 2, pp. 278-292, 2000.

[13] C. Lizama, "An operator theoretical approach to a class of fractional order differential equations," Applied Mathematics Letters, vol. 24, no. 2, pp. 184-190, 2011.

[14] C. Lizama and G. M. N’Guérékata, "Bounded mild solutions for semilinear integro differential equations in Banach spaces," Integral Equations and Operator Theory, vol. 68, no. 2, pp. 207227, 2010.

[15] M. Li, C. Chen, and F.-B. Li, "On fractional powers of generators of fractional resolvent families," Journal of Functional Analysis, vol. 259, no. 10, pp. 2702-2726, 2010.

[16] K. Li, J. Peng, and J. Jia, "Cauchy problems for fractional differential equations with Riemann-Liouville fractional derivatives," Journal of Functional Analysis, vol. 263, no. 2, pp. 476-510, 2012.

[17] G. M. Mophou and G. M. N’Guérékata, "Existence of mild solutions of some semilinear neutral fractional functional evolution equations with infinite delay," Applied Mathematics and Computation, vol. 216, no. 1, pp. 61-69, 2010.

[18] G. M. Mophou and G. M. N’Guérékata, “A note on a semilinear fractional differential equation of neutral type with infinite delay," Advances in Difference Equations, vol. 2010, Article ID 674630, 8 pages, 2010.

[19] J. Wang, Z. Fan, and Y. Zhou, "Nonlocal controllability of semilinear dynamic systems with fractional derivative in Banach spaces," Journal of Optimization Theory and Applications, vol. 154, no. 1, pp. 292-302, 2012.

[20] J. Wang, Y. Zhou, and M. Fečkan, "Abstract Cauchy problem for fractional differential equations," Nonlinear Dynamics, vol. 71, no. 4, pp. 685-700, 2013.

[21] L. Byszewski and V. Lakshmikantham, "Theorem about the existence and uniqueness of a solution of a nonlocal abstract Cauchy problem in a Banach space," Applicable Analysis, vol. 40, no. 1, pp. 11-19, 1991.

[22] L. Chen and Z. Fan, "On mild solutions to fractional differential equations with nonlocal conditions," Electronic Journal of Qualitative Theory of Differential Equations, no. 53, pp. 1-13, 2011.

[23] Z. Fan, "Existence of nondensely defined evolution equations with nonlocal conditions," Nonlinear Analysis, vol. 70, no. 11, pp. 3829-3836, 2009.

[24] Z. Fan, "Impulsive problems for semilinear differential equations with nonlocal conditions," Nonlinear Analysis, vol. 72, no. 2, pp. 1104-1109, 2010.

[25] Z. Fan and G. Li, "Existence results for semilinear differential equations with nonlocal and impulsive conditions," Journal of Functional Analysis, vol. 258, no. 5, pp. 1709-1727, 2010.

[26] E. Hernández, J. S. dos Santos, and K. A. G. Azevedo, "Existence of solutions for a class of abstract differential equations with nonlocal conditions," Nonlinear Analysis, vol. 74, no. 7, pp. 2624-2634, 2011.

[27] J. Liang, J. H. Liu, and T.-J. Xiao, "Nonlocal problems for integrodifferential equations," Dynamics of Continuous, Discrete \& Impulsive Systems, vol. 15, no. 6, pp. 815-824, 2008.

[28] J. Liang, J. H. Liu, and T.-J. Xiao, "Nonlocal impulsive problems for nonlinear differential equations in Banach spaces," Mathematical and Computer Modelling, vol. 49, no. 3-4, pp. 798-804, 2009. 
[29] J. Liang and Z. Fan, "Nonlocal impulsive Cauchy problems for evolution equations," Advances in Difference Equations, vol. 2011, Article ID 784161, 17 pages, 2011.

[30] X. M. Xue, "Existence of semilinear differential equations with nonlocal initial conditions," Acta Mathematica Sinica, vol. 23, no. 6, pp. 983-988, 2007.

[31] Y. Zhou and F. Jiao, "Nonlocal Cauchy problem for fractional evolution equations," Nonlinear Analysis, vol. 11, no. 5, pp. 44654475, 2010.

[32] L. Zhu and G. Li, "Existence results of semilinear differential equations with nonlocal initial conditions in Banach spaces," Nonlinear Analysis, vol. 74, no. 15, pp. 5133-5140, 2011.

[33] L. Zhu, Q. Huang, and G. Li, "Existence and asymptotic properties of solutions of nonlinear multivalued differential inclusions with nonlocal conditions," Journal of Mathematical Analysis and Applications, vol. 390, no. 2, pp. 523-534, 2012.

[34] J. Banaś and K. Goebel, Measures of Noncompactness in Banach Spaces, vol. 60 of Lecture Notes in Pure and Applied Mathematics, Marcel Dekker, New York, NY, USA, 1980. 


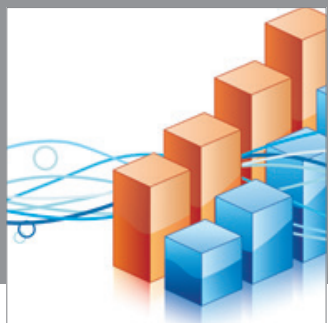

Advances in

Operations Research

mansans

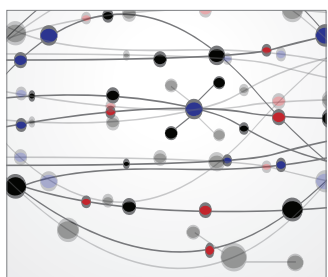

The Scientific World Journal
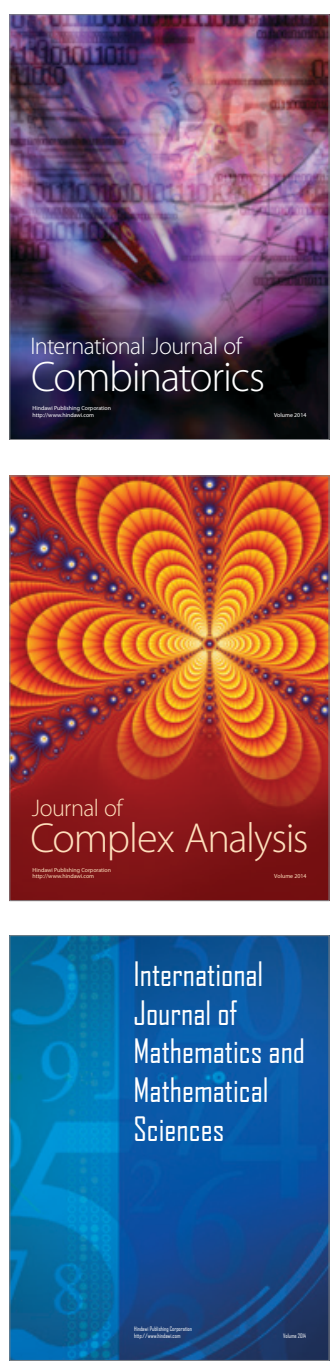
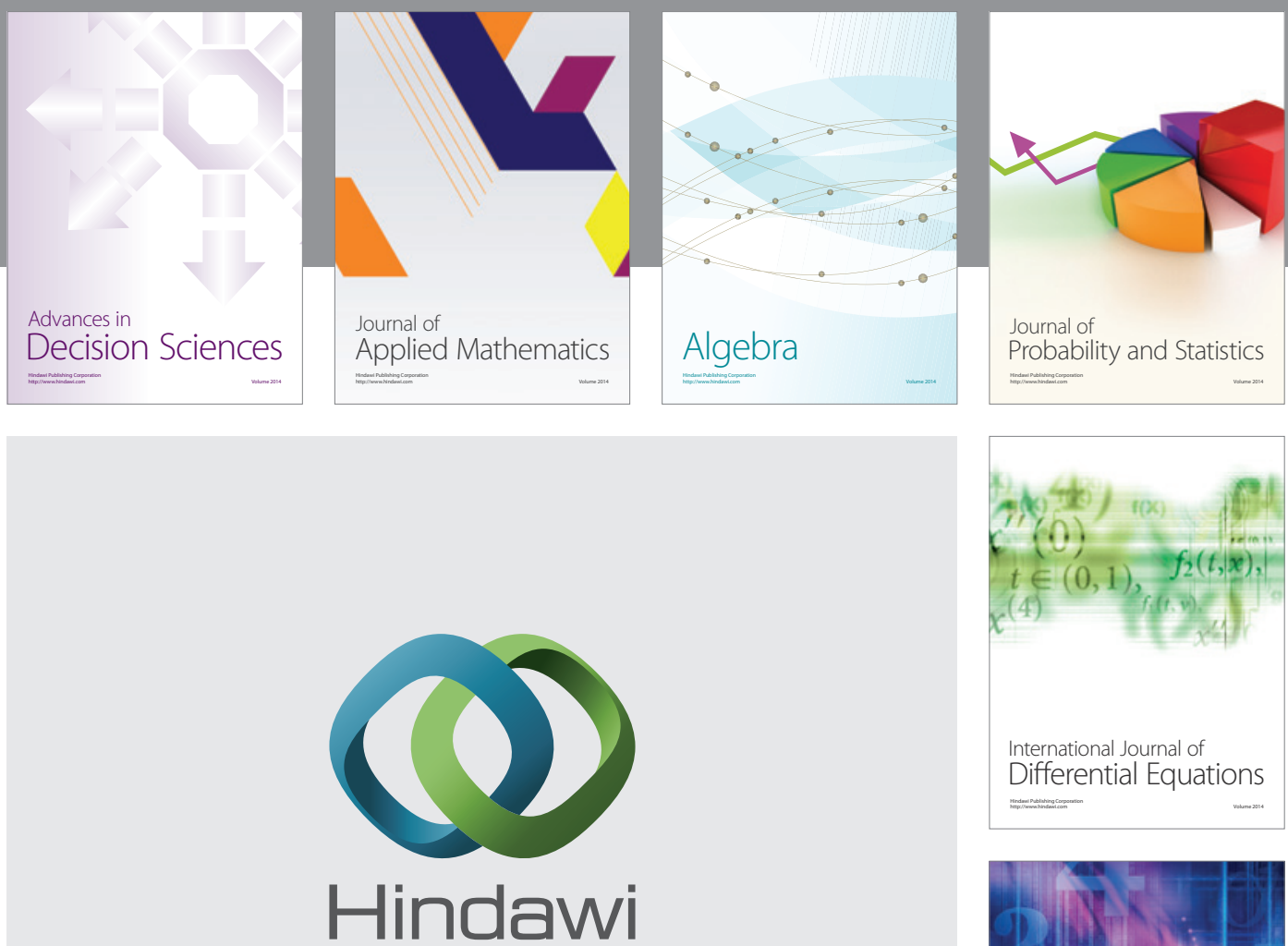

Submit your manuscripts at http://www.hindawi.com
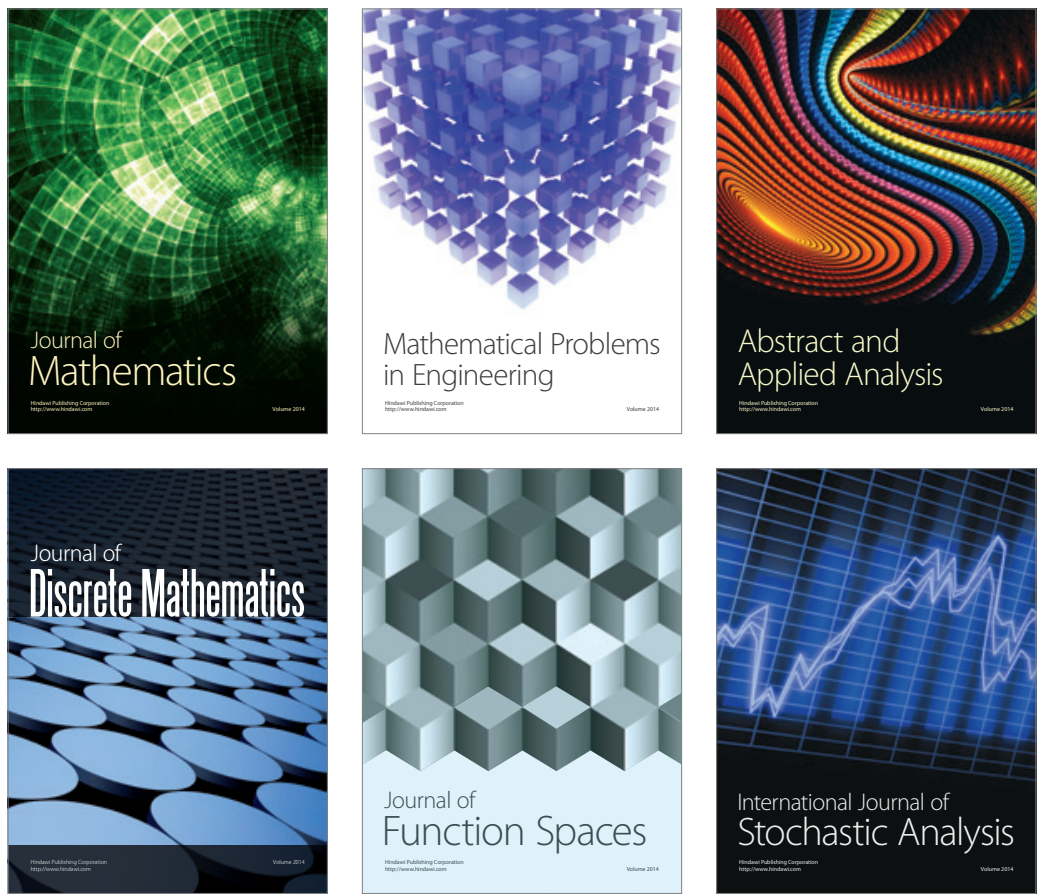

Journal of

Function Spaces

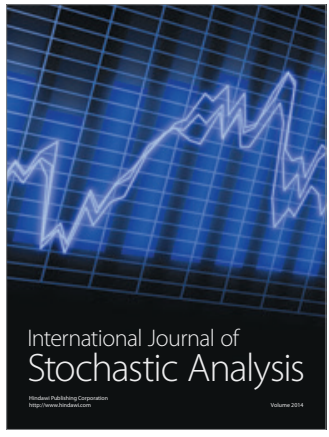

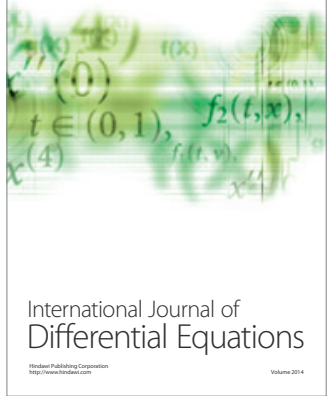
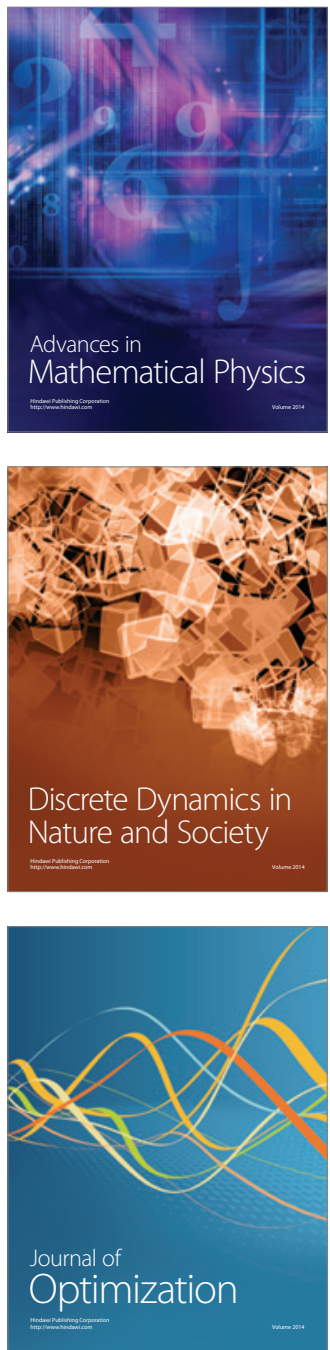